\title{
1 Thermal stress induces positive phenotypic and molecular feedback loops in 2 zebrafish embryos
}

3

4 Lauric Feugere ${ }^{1}$, Victoria F. Scott ${ }^{1}$, Quentin Rodriguez-Barucg ${ }^{2}$, Pedro Beltran-Alvarez ${ }^{2}$ \& Katharina C. 5 Wollenberg Valero ${ }^{1 *}$

6

$7 \quad{ }^{1}$ Department of Biological and Marine Sciences, University of Hull, Cottingham Road, Kingston upon Hull, HU6 8 7RX, United Kingdom.

$9{ }^{2}$ Department of Biomedical Sciences, University of Hull, Cottingham Road, Kingston upon Hull, HU6 7RX, 10 United Kingdom.

12 *Corresponding author: k.wollenberg-valero@hull.ac.uk, +44 (0)1482 465187

13 ORCID: 0000-0001-8858-1804 


\section{Abstract}

Aquatic organisms must cope with both rising and rapidly changing temperatures. These environmental changes can affect numerous traits, from molecular to ecological scales. Biotic stressors can induce the release of chemical cues which trigger behavioural responses in other individuals. In this study, we infer whether abiotic stressors, such as fluctuating temperature, may similarly propagate stress responses between individuals in fish not directly exposed to the stressor. To test this hypothesis, zebrafish (Danio rerio) embryos were exposed for 24 hours to fluctuating thermal stress, to medium in which another embryo was thermally stressed before ("stress medium"), and to a combination of these. Growth, behaviour, and expression of a panel of genes were used to characterise the thermal stress response and its propagation between embryos. Both high temperatures and stress medium significantly accelerated development and altered embryonic behaviour. Thermal stress significantly decreased the expression of the antioxidant gene SOD1, eight hours after the end of exposure. Of note, we found that the expression of sulfide:quinone oxidoreductase (SQOR), likewise a part of the antioxidant metabolism relevant in vertebrate stress response, and of interleukin-1 $\beta$ (IL-1 $\beta$ ), involved in the immune response, were significantly altered by stress medium. This study illustrates the existence of positive thermal stress feedback loops in zebrafish embryos that induce stress in conspecifics. This evidence that thermal stress due to fluctuating, high temperatures can be propagated may be relevant for species found in high densities, either in aquaculture or in the natural environment, in a context of global change.

Keywords: behaviour, chemical communication, climate warming, gene expression, feedback loop, thermal stress, zebrafish embryos

\section{Introduction}

Temperature is the abiotic 'ecological master factor' regulating the biology of ectotherms (Brett, 1971; LópezOlmeda \& Sánchez-Vázquez, 2011). Ectotherms face thermal cycles that shape their biological rhythms by modulating survival, growth, and by triggering irreversible changes on thermal tolerance ranges of adults (Colinet et al., 2015; Kingsolver et al., 2015; Lim et al., 2017; López-Olmeda \& Sánchez-Vázquez, 2011; Schaefer \& Ryan, 2006). Those natural, regular thermal fluctuations experienced during early development are necessary to maximise fitness-relevant traits and thermal tolerance (Hall \& Warner, 2020b; Kingsolver et al., 2015; Lim et al., 2017; Schaefer \& Ryan, 2006). Besides higher average temperatures, global warming will be characterised by both greater thermal variability and more extreme thermal events of longer duration and magnitude (Pörtner et al., 2019; Vasseur et al., 2014). These altered thermal rhythms may pose higher risks to species than simply higher mean temperatures, due to the asymmetry of the thermal fitness curve (Colinet et al., 2015; Saxon et al., 2018; Vasseur et al., 2014). Here, we exposed zebrafish embryos to several peaks of higher temperatures that consisted in thirteen exposures to $+5^{\circ} \mathrm{C}$ within 24 hours. Although this thermal regime departs from natural, environmental 
relevant extreme heatwaves, it serves to model the effects of repeated thermal stress upon the stress response of zebrafish embryos.

Fish as ectothermic vertebrates are susceptible to changes in the thermal environment, particularly to higher temperatures close to their upper thermal limits (Araújo et al., 2013; I. J. Morgan et al., 2001; Paaijmans et al., 2013). Early developmental stages have narrower thermal ranges than adults (Skjærven et al., 2014). Temperature regimes during development have irreversible effects as they modulate subsequent stages, making early fish embryos vulnerable or "bottleneck" stages in the context of climate change (Pörtner \& Peck, 2010; Scott \& Johnston, 2012; Villamizar et al., 2012). Examples for persistent effects of temperature changes during development may involve alterations of swimming performance and cardiac anatomy (Dimitriadi et al., 2018), masculinisation (Ribas et al., 2017) and increased mortality (Hosseini et al., 2019), which can shape the future trajectory of populations. Altogether, this illustrates a rising concern about the response of fish to global change, particularly at early stages of development.

The zebrafish (Danio rerio) is now emerging as a model organism to study the effects of thermal stress, including at the molecular level (Brown et al., 2015; Clark et al., 2011; Long et al., 2012; Luu et al., 2020). Adult zebrafish are eurythermal, naturally tolerating a wide range of temperatures $\left(16.5-38.6^{\circ} \mathrm{C}\right)$ with optimal temperature around $27-28.5^{\circ} \mathrm{C}$. They may face natural thermal variations of $\sim 5^{\circ} \mathrm{C}$ daily, and from 6 to $38^{\circ} \mathrm{C}$ seasonally (Engeszer et al., 2007; López-Olmeda \& Sánchez-Vázquez, 2011; Spence et al., 2008). However, early stages of zebrafish only tolerate minimum temperatures of $22-23^{\circ} \mathrm{C}$ and maximum temperatures of around $32^{\circ} \mathrm{C}$ to develop normally (Pype et al., 2015; Schirone \& Gross, 1968; Schnurr et al., 2014). Warm-adapted species such as the zebrafish that are living near their upper thermal limit may be among the 'losers' of climate change (Somero, 2010). Of note, the thermal biology of zebrafish is conserved in laboratory populations, in spite of laboratory domestication, which makes them an adequate model organism to investigate the effects of climate change in the laboratory (Brown et al., 2015; R. Morgan et al., 2019).

Besides the above-mentioned effects on sex ratio, direct mortality and adult phenotype, responses to thermal stress at the early stages of development involve altered behaviour, developmental rate, and altered gene expression related to the physiological stress response. Behavioural thermoregulation is one major thermoregulatory process in ectotherms (López-Olmeda \& Sánchez-Vázquez, 2011). This is evident from a tightly controlled thermotaxis response in response to heat in zebrafish larvae (Haesemeyer et al., 2015, 2018). Heat stressed zebrafish larvae display more anxiety-like behaviours (Bai et al., 2016) and transient hyperactivity (Yokogawa et al., 2014). High temperatures also accelerate the embryonic development in zebrafish embryos as shown by the 2.8 -fold increase in somitogenesis frequency across a $20-30^{\circ} \mathrm{C}$ range or the twice faster development at $33^{\circ} \mathrm{C}$ compared to $26^{\circ} \mathrm{C}$ (Hallare et al., 2005; Long et al., 2012; Schröter et al., 2008). Repeated exposure to sublethal temperatures, however, may depress development (Hall \& Warner, 2020a). At the molecular level, heat stress leads to numerous molecular effects from the accumulation of reactive oxygen species (ROS) (Madeira et al., 2016; Vinagre et al., 2012) to changes in global gene expression patterns (Logan \& Buckley, 2015; Long et al., 2012; Ribas et al., 2017) along the hypothalamic-pituitary-interrenal (HPI) axis (Alsop \& Vijayan, 2009). For example, Cu/Zn-superoxide 
dismutase I (SOD1) neutralises oxygen radicals to protect cells from oxidative stress (Cheng et al., 2018; Wang et al., 2016), and is heat inducible in zebrafish embryos (Icoglu Aksakal \& Ciltas, 2018). Another less well studied enzyme, sulfide:quinone oxidoreductase (SQOR) is upregulated by heat stress in half-smooth tongue sole (Cynoglossus semilaevis) (Guo et al., 2016) and by hypoxia in Nile tilapia (Oreochromis niloticus) (J. H. Xia et al., 2018). SQOR expression is co-induced by both cold stress and hypoxia in zebrafish embryos (Long et al., 2012, 2015). The innate immune system is challenged by both high temperature and thermal fluctuations in teleost early stages (Mariana \& Badr, 2019; Zhang et al., 2018). For instance, interleukin-1 $\beta$ (IL-1 $\beta$ ) is upregulated in zebrafish raised at high temperature but experiencing an immune challenge at low temperature (Zhang et al., 2018) and in zebrafish embryos exposed to high temperature (Icoglu Aksakal \& Ciltas, 2018). This gene plays a central role in the stress response, having neuromodulatory and behavioural functions (Goshen \& Yirmiya, 2009; Metz et al., 2006; Vitkovic et al., 2000).

Largely overlooked to date, however, is the question of whether the response to thermal stress can be transmitted to other individuals. Fish use chemical communication to alert others of a threat using so-called 'alarm cues' (released after skin damage) or 'disturbance cues' (released without injury following a biotic stressor) (Jordão \& Volpato, 2000). Exposure to conspecific predation-related disturbance cues induces stress-like responses in several fish species (Barcellos et al., 2011, 2014; Bett et al., 2016; Ferrari et al., 2008; Jordão \& Volpato, 2000; Toa et al., 2004), including zebrafish (Barcellos et al., 2014). Importantly, even fish early stages are capable of such chemical communication as they respond to alarm cues (Atherton \& McCormick, 2015, 2017; Oulton et al., 2013; Poisson et al., 2017). Despite the wealth of information on biotic stress-induced stress propagation, this phenomenon is only known from a few abiotic stressors, such as low pH, acute fasting or handling (Abreu et al., 2016; Feugere et al., in review), but in response to heat is only known from crayfish (Hazlett, 1985). Stress induces the release of metabolites into the environment, including hormones of the HPI axis (Barcellos et al., 2014; McGlashan et al., 2012), $\mathrm{CO}_{2}$ (McGlashan et al., 2012), respiratory byproducts, catecholamines, or nitrogenous metabolic products such as urea (Bairos-Novak et al., 2017; Giaquinto \& Hoffmann, 2012; Henderson et al., 2017; Hubbard et al., 2003), but less is known about their effects on communication. Distinct from alarm and disturbance cues, we introduced the term 'stress metabolite' referring to such cues induced without injury as a byproduct of the response to abiotic stress (Feugere et al., in review).

In this contribution we infer whether thermal stress can be propagated to naive receivers. To test this hypothesis, zebrafish embryos were exposed to independent and combined treatments of thermal stress and medium putatively containing stress metabolites induced by this thermal stress. Zebrafish embryo behaviour, growth rate, and expression of genes involved in the immune response (IL-1 $\beta$ ) and antioxidant pathways (SOD1, SQOR) were investigated. We expected that (i) fluctuating high temperatures, similar to constant higher temperatures, trigger developmental, behavioural and molecular stress responses in zebrafish embryos. Second (ii), we hypothesised that these responses could induce a positive feedback loop in naive receiver embryos, which are however (iii) not elicited by non-stress metabolites. It should be emphasised that our work primarily aimed to investigate the 
existence of stress propagation induced by repeated thermal stress, in a context of growing concern for a future more stressful environment, rather than study the effects of realistic heatwaves.

\section{Materials and Methods}

\section{Experimental design}

124 For detailed zebrafish husbandry and breeding methods, see Supplementary Information. Just before the beginning 125 of experimental treatments, several zebrafish embryos per clutch were photographed to estimate the median initial 126 stage. Exposure began around the blastula stage (median stage: $2.75 \mathrm{hpf}$, at 512-cells). Fertilised healthy embryos 127 (with chorion) were selected and individually placed into $0.2 \mathrm{~mL} 8$-strip PCR tubes prefilled with $200 \mu \mathrm{L}$ of $1 \mathrm{X}$ 128 E3 medium.

129 In a two-factorial design, embryos were exposed to different combinations of the two factors, temperature stress 130 and temperature-induced stress medium. For this purpose, embryos were exposed to either thermal stress or control temperature protocols within a thermocycler, in either fresh medium or medium where embryos had previously been exposed to thermal stress and containing putative stress metabolites. All experimental treatments are detailed in Figure 1 and Table S1. The thermal stress protocol spanned $16.25 \mathrm{hrs}$, divided into thirteen 75 -min series of temperature fluctuations between $27,29,32,29$, and $27^{\circ} \mathrm{C}$, with each temperature step being maintained for 15 min. Thermal stress mimicked $+5^{\circ} \mathrm{C}$ temperature peaks over zebrafish optimal temperature (a total of $\mathrm{n}=13$ peaks) reaching the sublethal temperature of $32^{\circ} \mathrm{C}$ (Scott \& Johnston, 2012). A recovery time of $7.75 \mathrm{hrs}$ at $27^{\circ} \mathrm{C}$ followed the fluctuating temperatures period to reach a total incubation time of $24 \mathrm{hrs}$. The control thermal protocol was a steady $27^{\circ} \mathrm{C}$ for $24 \mathrm{hrs}$. E3 media following control or thermal stress conditions were reused for 'control medium' and 'stress medium' treatments, respectively. In total this yielded five treatments: control (C, control protocol with fresh medium), control medium (CM, control protocol with reused medium from C), thermal stress (TS, thermal stress protocol with fresh medium), stress medium (SM, control protocol with reused medium from TS), and thermal stress + stress medium (TS+SM, thermal stress protocol with reused medium from TS). Used media were immediately re-used for treatments containing putative stress or control metabolites, respectively. As development in zebrafish accelerates with higher temperature (Kimmel et al., 1995) but decelerates with darkness (Bucking et al., 2013; Villamizar et al., 2014), additional control embryos were monitored after longer incubation times: 31 hrs (C31), $37 \mathrm{hrs} \mathrm{(C37),} \mathrm{and} 46 \mathrm{hrs}$ (C46). These times were adjusted for darkness-raised embryos to reach the stages of prim-6 (25 hpf), prim-16 (31 hpf), and late pharyngula (35 - $42 \mathrm{hpf}$ ), respectively. Initial, final, and total exposure times were used to standardise each procedure. After incubation, transparent embryos were deemed alive and kept for subsequent steps. Before and after each exposure, embryo media were sampled to measure $\mathrm{pH}$ and $\mathrm{O}_{2}$ saturation levels that could impact embryos in the used media. Oxygen levels were averaged from 1-2 min measurements using a glass vial equipped with an oxygen sensor spot (OXSP5, sensor code: SC7-538-193, 152 Pyroscience $\mathrm{GmbH}$ ) and connected to the FireSting $\mathrm{O}_{2}$ Fiber Optic Oxygen Meter (FSO2-4, Pyroscience GmbH) 153 and Oxygen Logger software (Pyroscience $\mathrm{GmbH}$ ). Constant medium $\mathrm{pHs}$ were verified using the Fisherbrand ${ }^{\mathrm{TM}}$ accumet $^{\mathrm{TM}} \mathrm{AB} 150 \mathrm{pH}$ Benchtop Meters. 


\section{Analysis of phenotypic data}

157 Phenotypic analyses were conducted for all eight treatments. Embryos were placed in a watch glass vial and videoed by small batches using the camera setup (see Supplementary Information) over 15-30 seconds. Embryos were placed under light (similar intensity across measurements) to elicit a startle-like response after exposure in darkness. Behavioural data was analysed using Danioscope (Noldus). When possible, several videos were recorded for each embryo clutch and behavioural measurements were averaged for each individual embryo. Analysis of behavioural responses were conducted on the video-length standardised burst activity percentage (percentage of the time - from the total measurement duration - the embryo was moving). Final embryonic stages were estimated following Kimmel et al. (1995) from several photographs of embryos within their chorions using the criteria somite number, yolk extension to yolk ball ratio, presence and morphology of otoliths, tail aspect, presence of lens primordium, presence and position of the cerebellum relatively to the eyes, and pigmentation. The growth index was calculated as in equation 1 .

Statistical analyses were conducted in RStudio (RStudio Team, 2020). Outliers among behavioural values were excluded from statistical analyses using Tukey's method with a 1.5 interquartile range cut-off. First, the effects of stress medium and thermal stress predictors across the C, TS, SM, and TS+SM treatments were evaluated. ShapiroWilk and Levene's tests were used to evaluate normality and homogeneity of variances, respectively. Normalisation methods were compared using the BestNormalize R package (Peterson \& Cavanaugh, 2020). The growth index was parameterised using an order normalising transformation (Shapiro-Wilk's $\mathrm{P}=0.98$, Levene's $\mathrm{P}$ $=0.27$ ) and analysed by a two-way ANOVA with thermal stress and stress medium as predictors, followed by post-hoc pairwise Student's t-tests between control C and each experimental condition. The burst activity percentage and final stage (in hpf) could not be normalized and were analysed for the effects of both predictors using nonparametric Scheirer-Ray-Hare tests from the rcompanion R package (Mangiafico, 2018), followed by pairwise post-hoc Wilcoxon-Mann-Whitney tests. The final embryonic developmental period was coded as segmentation or pharyngula and analysed for the effects of thermal stress, stress medium as factors and initial stages as covariate using a generalised logistic model followed by pairwise post-hoc comparisons using the emmeans R package (Lenth, 2019).

183 Additional pairwise comparisons were performed of all response variables of CM against C and SM. To determine whether behavioural effects of treatment were related to developmental acceleration, pairwise comparisons of behaviour of control embryos (C) were compared to that of older control embryos (C31, C37, C46) using KruskalWallis tests. Fourth, burst activity percentages of embryos from stress medium and thermal stress treatments were compared against that of control embryos incubated for longer times using pairwise Wilcoxon-Mann-Whitney tests. Multiple comparisons were corrected using Bonferroni adjustments. Cohen's $|\mathrm{d}|$ were obtained from the 
190

191

192

193

194

195

196

197

198

199

200

201

202

203

204

205

206

207

208

209

210

211

212

213

214

215

216

217

218

219

220

221

222

223

224

225

or according to Lenhard \& Lenhard (2016), respectively. Effect size was qualified based on thresholds given in Sawilowsky (2009): very small: $|\mathrm{d}|>0.01$, small: $|\mathrm{d}|>0.2$, medium: $|\mathrm{d}|>0.5$, large: $|\mathrm{d}|>0.8$, very large: $|\mathrm{d}|>1.20$, huge: $|\mathrm{d}|>2.0$.

\section{Gene expression}

Gene expression analyses were conducted for CM, C, SM, TS, and TS+SM treatments $(\mathrm{n}=3$ pooled biological replicates of 60 embryos per treatment). Embryos were snap-frozen at $-80^{\circ} \mathrm{C}$ immediately after experimental treatments. Total RNA was extracted using a High Pure RNA isolation kit (Sigma-Aldrich) following the manufacturer's recommendations. cDNA was synthesized using Superscript II $^{\mathrm{TM}}$ Reverse Transcriptase (Invitrogen, Life Technologies Ltd.) with sample randomisation. TaqMan ${ }^{\circledR}$ Gene Expression Assays (ThermoFisher Scientific) and 2X qPCR Bio Probe Hi-ROX (PCRBiosystems) were used to quantify the expression of three genes of interest (SQOR, SOD1, and IL-1 $\beta$ ) normalised to two reference genes (ef1-a, $\beta$-actin). The effects of stress medium and thermal stress on the $\log _{2} 2^{-\Delta \Delta C T}\left(\log _{2}\right.$ fold-change) values were investigated using the eBayes and lmFit functions within the limma package (Ritchie et al., 2015) within the Bioconductor v.3.11 (Ihaka \& Gentleman, 1996) R environment. Next, pairwise post-hoc comparisons on C versus SM, TS, or $\mathrm{TS}+\mathrm{SM}$, and $\mathrm{CM}$ versus $\mathrm{C}$ or $\mathrm{CM}$ were performed using pairwise moderated t-tests with Bonferroni adjustments. Effect sizes (Cohen's $|\mathrm{d}|$ ) were calculated as above. More details on the gene expression analysis are given as Supplementary Information.

\section{Results}

\section{Phenotypic effects of thermal stress and its propagation}

First, the phenotypic effects of fluctuating thermal stress and of stress medium treatments were analysed. Embryonic growth indices were significantly accelerated by stress medium (small effect size, $\mathrm{F}=6.291, \mathrm{P}=$ 0.0128), thermal stress (large effect size, $F=75.502, \mathrm{P}<0.0001$ ), and their combination (very large effect size, $\mathrm{F}$ $=7.498, \mathrm{P}=0.0067$, Figure 2a, Table 1). Post-hoc tests revealed that TS $(\mathrm{t}=-7.9874, \mathrm{P}<0.0001), \mathrm{SM}(\mathrm{t}=-$ 3.6784, $\mathrm{P}=0.0012)$, and the combined treatment TS+SM $(\mathrm{t}=-7.2413, \mathrm{P}<0.0001)$ all accelerated growth, compared to the control C (Table S2). The growth acceleration was accompanied by a median advancement in embryonic stages of 3 to 9 hours, resulting in a switch from the segmentation to the pharyngula stage, compared to controls C and CM (see Supplementary Information, Figure S1a-b, Tables S3-S4). Treatments had no obvious effect on mortality.

We reasoned that any observed effects of stress medium may be due to regularly excreted metabolites, which accumulate towards the end of each treatment independently of thermal stress and would be up-concentrated in SM and TS+SM treatments. This warranted the use of an additional control, the 'control medium' (CM), that is, medium that had previously hosted control embryos. This control helped us exclude oxygen saturation and $\mathrm{pH}$ as confounding effects, which were also independently measured at the beginning of treatments and in all cases fell within zebrafish natural tolerance ranges (Strecker et al., 2011; Zahangir et al., 2015). We therefore assessed 
whether stress medium, that is, medium where embryos had been exposed to thermal stress, evoked different effects compared to control medium. Embryonic growth in CM was compared to growth in C and SM. Embryos in CM grew slightly slower than control embryos $(\mathrm{t}=2.3472, \mathrm{P}=0.0418)$ and much slower than embryos in $\mathrm{SM}$ $(\mathrm{t}=-6.7215, \mathrm{P}<0.0001$, Figure 2a, Table S2).

230 Next, we investigated the behavioural startle-like response to light (Figure $2 b$ ). Burst activity percentages were significantly lowered by both predictors stress medium $(\mathrm{H}=9.3222, \mathrm{P}=0.0023)$ and thermal stress $(\mathrm{H}=17.008$, $\mathrm{P}<0.0001)$, whereas the interaction term was not significant $(\mathrm{H}=1.8193, \mathrm{P}=0.1774$, Table 2). Post-hoc comparisons showed that embryos treated with $\mathrm{SM}(\mathrm{W}=1,387, \mathrm{P}=0.0018)$, TS $(\mathrm{W}=3,548, \mathrm{P}=0.0003)$, and $\mathrm{TS}+\mathrm{SM}(\mathrm{W}=2,455, \mathrm{P}<0.0001)$ all displayed lower burst percentages compared to control C. Embryos exposed to $\mathrm{CM}$ showed even stronger decline in burst activity percentages compared to $\mathrm{C}(\mathrm{W}=3,287, \mathrm{P}<0.0001)$ and $\mathrm{SM}$ (W = 1,745, $\mathrm{P}<0.0001$, Table S5).

Considering the aforementioned thermal stress-induced growth acceleration, stage-dependent effects of behaviour were investigated in control embryos incubated for $24,31,37$, or $46 \mathrm{hrs}$. We observed that burst activity percentages significantly decreased with development in pre-hatching control embryos raised for 31, 37, and 46 hrs compared to those incubated for $24 \mathrm{hrs}(\mathrm{P}<0.0001$, Figure S2c, Table S6). However, neither final stages $(\mathrm{P}=$ $0.1627)$ nor growth index $(\mathrm{P}=0.5027)$ were correlated with burst activity percentages across treated embryos (Table S5). Moreover, pairwise tests showed that stressed embryos under development accelerated by TS, SM, and TS+SM treatments were significantly more active than control embryos reaching the same median stage of prim-6 in $\mathrm{C} 31$ ( $\mathrm{P}<0.0001$, Figure 2b, Table S7).

\section{Effects of thermal stress and its propagation on stress response-related gene expression}

The whole-embryo expression of three stress-inducible candidate genes (IL-1 $\beta$, SOD1 and SQOR) was analysed (Figure 3, Tables 3 and S8). Thermal stress was not a significant predictor for the expression of either IL-1 $\beta$ or SQOR, but SOD1 expression was lower in the TS treatment compared to the control C (very large effect size but marginal significance, $\mathrm{t}=2.76, \mathrm{P}=0.045$ ). In contrast, stress medium significantly reduced $\mathrm{IL}-1 \beta$ expression (very large effect size, $\mathrm{t}=2.28, \mathrm{P}=0.038$ ) and increased SQOR expression levels (huge effect size, $\mathrm{t}=-3.54, \mathrm{P}=0.003$ ), but had no effects on SOD1 expression.

Pairwise tests revealed that SOD1 gene expression in control medium (CM) was much lower compared to C (huge all three studied markers. SM significantly reduced the expression of IL-1 $\beta$ (very large effect size, $\mathrm{t}=2.62, \mathrm{P}=$ 0.034 ) and increased that of SOD1 (very large effect size, $t=-2.64, \mathrm{P}=0.034$ ), whereas there was a only a nonsignificant trend towards increased SQOR expression $(\mathrm{P}=0.08)$. Relative to the control, the gene expressions of IL1- $\beta$ and SQOR in SM showed opposite patterns compared to CM. Taken together, our gene expression results suggest that incubation in stress medium leads to reduced IL-1 $\beta$ expression and increased SQOR expression. 


\section{Discussion}

\section{Fluctuating high temperatures induce a stress response in zebrafish embryos}

264 Communication between conspecifics can potentiate the response of a group to stressors (Giacomini et al., 2015), consequently the combined effects of abiotic stressors and any feedback loops they induce are of great importance in the context of global change. For instance, by the end of the century not only temperature but also chemical cues associated with a warming aquatic environment could be altered (Chivers et al., 2013; Lienart et al., 2016; Roggatz et al., 2019), and warming is known to affect predator-prey communication via infochemicals (J. Xia et al., 2020). The overarching aim of this work was to investigate whether zebrafish embryos can propagate aspects of their response to fluctuating heat stress to naive receiver embryos through positive feedback loops. First, we investigated the effects of fluctuating thermal stress on zebrafish embryos. Our results showed that heat stressed embryos grew faster than control embryos, which is consistent with previous reports (Long et al., 2012; Schnurr et al., 2014). This may in turn favour premature hatching of smaller larvae (Cingi et al., 2010; Schmidt \& Starck, 2010). Stressed embryos were less active than control embryos incubated for 24 hours but were hyperactive compared to controls developed to the same stage of prim-6 without stress treatments. Our results support previous observations that heat stress triggers higher behavioural activity in zebrafish early stages (Gau et al., 2013; Yokogawa et al., 2014). Such behavioural alterations may be explained by (i) energy trade-offs between behaviour, growth, and the metabolic costs of stress response (Barton \& Iwama, 1991; Simčič et al., 2015), as well as (ii) temperature-dependent molecular changes in gene expression, epigenetic gene regulation, or post-translational modification related to behaviour, potentially involving circadian clock and neurodevelopmental genes (Colson et al., 2019; López-Olmeda \& Sánchez-Vázquez, 2011).

At the gene expression level, we found that IL-1 $\beta$ and SQOR remained unchanged in thermally-stressed embryos. al., 2016; Long et al., 2012, 2015; J. H. Xia et al., 2018) and of heat-induced increased IL-1 $\beta$ levels in adult black rockfish (Sebastes schlegelii) (Lyu et al., 2018) and zebrafish embryos (Icoglu Aksakal \& Ciltas, 2018). Higher temperature stress usually triggers an upregulation of SOD1 (Liu et al., 2018; Mahanty et al., 2016). In contrast, fluctuating thermal stress in this study reduced SOD1 expression compared to the control, measured almost eight hours after the cessation of thermal fluctuations. These inverted gene expression patterns under fluctuating, as compared to constant thermal stress, might be related to energetic depletion as a result of the thermal cycles (Schaefer \& Ryan, 2006). Repetition of heat stress also downregulated heat shock proteins in lake whitefish (Coregonus clupeaformis) embryos (Whitehouse et al., 2017). Nevertheless, it should be acknowledged that the differences in developmental rate observed under fluctuating heat stress may superimpose confounding effects to the effects of the heat stimulus, as gene expression varies with ontogeny during zebrafish embryogenesis (Mathavan et al., 2005). Nonetheless, it is likely that fluctuating temperatures experienced through early development of zebrafish embryos cause lasting developmental and behavioural changes, similar to those already shown to occur in vertebrate ectotherms after static increased temperatures. 


\section{Thermal stress induces positive stress feedback loops in naive receiver embryos}

299

300

301

302

303

304

305

306

307

308

309

310

311

312

313

314

315

316

317

318

319

320

321

322

323

324

325

326

327

328

329

330

331

332

333

It is well-known that animals can chemically communicate a state of distress to others, although predation stress has traditionally received the most attention (Barcellos et al., 2014; Jordão \& Volpato, 2000). Here, we found that fluctuating thermal stress negatively affects naive conspecifics, with a similar directionality of effects than the thermal stress itself, which can be described as a positive feedback loop.

First, embryos in our experiment grew faster when subjected to stress medium obtained from previously heatstressed embryos, reaching similar stages to those of heat-stressed embryos. Such "catch-up" synchronous growth has been shown in egg-clustered embryos of several species and indicates the presence of embryo-embryo communication. Usually this communication serves to maximise energy costs against potential threats and to potentially facilitate group emergence (Aubret et al., 2016; Colbert et al., 2010; McGlashan et al., 2012).

Second, stress medium triggered behavioural hyperactivity compared to control embryos reaching an equivalent stage (prim-6). These results are in agreement with higher activities in rainbow trout embryos facing alarm cues (Poisson et al., 2017) but depart from lower behaviour activities in 24 hpf zebrafish embryos exposed to conspecific alarm cues (Lucon-Xiccato et al., 2020), which suggest that the response depends on the type of the cue and the tested model. In our experiment, these behavioural responses were measured, for the first time, as a response to temperature stress-elicited cues. Behavioural alteration was also observed in adult zebrafish in response to low $\mathrm{pH}$ and fasting stress-induced metabolites (Abreu et al., 2016), and adult marine invertebrates experiencing metabolites from low pH-stressed conspecifics and heterospecifics (Feugere et al, in review).

Third, stress medium originating from thermally-stressed embryos induced changes in gene expression patterns in naive conspecific receiver embryos. IL-1 $\beta$ was significantly downregulated in stress medium treatments. The stress medium-mediated inhibition of IL- $1 \beta$ in our experiment suggests that one of its inhibitor stress hormones, such as cortisol, adrenaline and the adrenocorticotropic hormone (Castillo et al., 2009; Castro et al., 2011), or HSF1 (Cahill et al., 1996) could be upregulated by stress media. Intriguingly, the expression of immune genes may be associated with behavioural changes in zebrafish, since highly responsive fish also have higher IL-1 $\beta$ expression (Kirsten et al., 2018). This indicates a functional link between the concomitant decreases of IL-1 $\beta$ and lower activity of stress medium-treated embryos compared to 24 hrs-incubated medium control embryos.

On the other hand, SQOR expression was significantly upregulated by stress medium. SQOR has been little studied in an environmental stress response context thus far, but emerged as a novel candidate marker from recent transcriptomics studies of thermal and oxidative stress (Guo et al., 2016; Long et al., 2012, 2015; Wollenberg Valero et al., 2019; J. H. Xia et al., 2018). SQOR is involved in the metabolism of hydrogen sulfide $\left(\mathrm{H}_{2} \mathrm{~S}\right)$, concentrations of which are toxic at supraphysiological levels, by tuning its neuromodulatory and biological roles (Augustyn et al., 2017; Chao et al., 2012; Horsman \& Miller, 2016; Jackson et al., 2012; Rose et al., 2017). Interestingly, SQOR and $\mathrm{H}_{2} \mathrm{~S}$ may be involved in the response to oxidative stress through increasing glutathione levels (Kimura et al., 2010; Yonezawa et al., 2007) and by mediating the antioxidant effects of CoQ10 (Kleiner et al., 2018). There is evidence that SQOR maintains ATP production (Quinzii et al., 2017) and has been proposed as a growth-related candidate gene (Zhuang et al., 2020). Kleiner and colleagues (Kleiner et al., 2018) found that 
an increase of SQOR may prevent oxidative stress by facilitating the antioxidant effects of CoQ10. Reversely, a downregulation of SQOR may reflect deficiency of its coenzyme CoQ10, which in turn alters the sulfide metabolism leading to accumulated $\mathrm{H}_{2} \mathrm{~S}$ levels and depletion of glutathione, that may cause oxidative damages (Luna-Sánchez et al., 2017; Quinzii et al., 2017; Ziosi et al., 2017). Therefore, the upregulation of SQOR under stress medium treatment could have multiple functions, from metabolising toxic levels of $\mathrm{H}_{2} \mathrm{~S}$ to restoring both ATP and GSH levels in response to stress communication from a conspecific. Altogether, our results indicate impaired immune and antioxidant responses in embryos exposed to propagated thermal stress.

\section{Stress medium and control medium do not induce similar feedback mechanisms}

Embryos exposed to the control medium developed slower than control embryos from E3 medium, showing opposite directionality to all other treatments. This opposite directionality was mirrored by the expression patterns of the three investigated genes. The behavioural response to stress medium was also significantly less pronounced than that to control medium, corroborating previous studies where metabolites from undisturbed versus stressed donors induced different responses in several species (Bairos-Novak et al., 2017; Bett et al., 2016). However, activity was much higher in control embryos from E3 medium than in embryos incubated in control medium. This finding may indicate that behavioural activity of zebrafish embryos is tightly controlled by the nature of their chemical environment (vs. relaxed in the absence of any cues), lending additional support to chemical communication as a global change-relevant parameter.

Lastly, the similarity in development and behavioural values in combined thermal stress plus stress medium treatments together with a larger effect size indicate that thermal stress and stress medium may excite similar molecular pathways regulating growth rate and behavioural activity, but that these pathways are saturated by single stressors, and cannot be further altered in the combined treatment. Conversely, our gene expression analysis revealed a difference in molecular responses between the two independent factors, thermal stress and stress medium. To better understand these contrasting synergistic vs. independent effects of thermal stress and stress medium, gene expression could be compared at global scale in future work.

\section{Conclusion}

To conclude, our study indicates that thermally-stressed zebrafish embryos induce a stress response in naive conspecifics that have not been exposed to thermal stress, from molecular to phenotypic level. This extends the concept of stress feedback loops and chemical communication of stress to fluctuating heat stress in a vulnerable life stage of fish. This is important because such stress feedback loops may be widespread and have implications on tipping points of ecosystems facing ongoing and future global change. We suggest further investigation into the identification of the nature of metabolites contained within stress medium, their molecular consequences at an individual level, as well as longer-term consequences for populations and ecosystems. 


\section{Acknowledgements}

The authors acknowledge Alan Smith and Sonia Jennings for providing fish care, Emma Chapman, Robert Donnelly for support in the laboratory, Adam Bates and Paulo Saldanha (University of Hull, UK) for support in discussing the experiments, and all members of the MolStressH2O research cluster (University of Hull, UK) for valuable discussions and peer review. For help with initial zebrafish method development we thank Antoinette Destefano, Amber Pinnock, Tiana Weeks, and Tia Rusciano (Bethune-Cookman University, USA) and Tyrese Taylor (Indiana University, USA). Funding was provided by the University of Hull to LF, VS, QRB, PBA and KWV and the Royal Society (RGS\R2\180033) to KWV.

\section{Conflicts of Interest}

The authors declare no conflicts of interest.

\section{Ethics approval}

All experiments were approved by the University of Hull Ethics committee under the approval U144b.

\section{Consent to participate}

All authors consented to participation.

\section{Consent for publication}

All authors consented to publication.

\section{Author contribution}

KWV conceived the study. KWV and PBA designed the experiments. LF, QRB, and VS collected the data and contributed to the statistical analysis. LF wrote the manuscript draft with PBA and KWV. All authors contributed to the final manuscript.

\section{References}

Abreu, M. S., Giacomini, A. C. V., Gusso, D., Koakoski, G., Oliveira, T. A., Marqueze, A., Barreto, R. E., \& Barcellos, L. J. G. (2016). Behavioral responses of zebrafish depend on the type of threatening chemical cues. Journal of Comparative Physiology A, 202(12), 895-901. https://doi.org/10.1007/s00359-016-1129-5

Alsop, D., \& Vijayan, M. M. (2009). Molecular programming of the corticosteroid stress axis during zebrafish development. Comparative Biochemistry and Physiology Part A: Molecular \& Integrative Physiology, 153(1), 49-54. https://doi.org/10.1016/j.cbpa.2008.12.008

Araújo, M. B., Ferri-Yáñez, F., Bozinovic, F., Marquet, P. A., Valladares, F., \& Chown, S. L. (2013). Heat freezes niche evolution. Ecology Letters, 16(9), 1206-1219. https://doi.org/10.1111/ele.12155 
403

404

405

406

407

408

409

410

411

412

413

414

415

416

417

418

419

420

421

422

423

424

425

426

427

428

429

430

431

432

433

434

435

436

437

438

Atherton, J. A., \& McCormick, M. I. (2015). Active in the sac: damselfish embryos use innate recognition of odours to learn predation risk before hatching. Animal Behaviour, 103, 1-6.

https://doi.org/10.1016/j.anbehav.2015.01.033

Atherton, J. A., \& McCormick, M. I. (2017). Kin recognition in embryonic damselfishes. Oikos, 126(7), 10621069. https://doi.org/10.1111/oik.03597

Aubret, F., Blanvillain, G., Bignon, F., \& Kok, P. J. R. (2016). Heartbeat, embryo communication and hatching synchrony in snake eggs. Scientific Reports, 6, 23519. https://doi.org/10.1038/srep23519

Augustyn, K. D. C., Jackson, M. R., \& Jorns, M. S. (2017). Use of Tissue Metabolite Analysis and Enzyme Kinetics To Discriminate between Alternate Pathways for Hydrogen Sulfide Metabolism. Biochemistry, 56(7), 986-996. https://doi.org/10.1021/acs.biochem.6b01093

Bairos-Novak, K. R., Mitchell, M. D., Crane, A. L., Chivers, D. P., \& Ferrari, M. C. O. (2017). Trust thy neighbour in times of trouble: background risk alters how tadpoles release and respond to disturbance cues. Proceedings of the Royal Society B: Biological Sciences, 284(1863). https://doi.org/10.1098/rspb.2017.1465

Bai, Y., Liu, H., Huang, B., Wagle, M., \& Guo, S. (2016). Identification of environmental stressors and validation of light preference as a measure of anxiety in larval zebrafish. BMC Neuroscience, 17(1), 63. https://doi.org/10.1186/s12868-016-0298-z

Barcellos, L. J. G., Koakoski, G., da Rosa, J. G. S., Ferreira, D., Barreto, R. E., Giaquinto, P. C., \& Volpato, G. L. (2014). Chemical communication of predation risk in zebrafish does not depend on cortisol increase. Scientific Reports, 4, 5076. https://doi.org/10.1038/srep05076

Barcellos, L. J. G., Volpato, G. L., Barreto, R. E., Coldebella, I., \& Ferreira, D. (2011). Chemical communication of handling stress in fish. Physiology \& Behavior, 103(3-4), 372-375. https://doi.org/10.1016/j.physbeh.2011.03.009

Barton, B. A., \& Iwama, G. K. (1991). Physiological changes in fish from stress in aquaculture with emphasis on the response and effects of corticosteroids. Annual Review of Fish Diseases, 1, 3-26. https://doi.org/10.1016/0959-8030(91)90019-G

Bett, N. N., Hinch, S. G., \& Yun, S.-S. (2016). Behavioural responses of Pacific salmon to chemical disturbance cues during the spawning migration. Behavioural Processes, 132, 76-84. https://doi.org/10.1016/j.beproc.2016.10.001

Brett, J. R. (1971). Energetic Responses of Salmon to Temperature. A Study of Some Thermal Relations in the Physiology and Freshwater Ecology of Sockeye Salmon (Oncorhynchus nerkd). American Zoologist, 11(1), 99-113. https://doi.org/10.1093/icb/11.1.99

Brown, A. R., Owen, S. F., Peters, J., Zhang, Y., Soffker, M., Paull, G. C., Hosken, D. J., Wahab, M. A., \& Tyler, C. R. (2015). Climate change and pollution speed declines in zebrafish populations. Proceedings of the National Academy of Sciences, 112(11), E1237-E1246. https://doi.org/10.1073/pnas.1416269112

Bucking, C., Lemoine, C. M. R., \& Walsh, P. J. (2013). Waste nitrogen metabolism and excretion in zebrafish 
embryos: effects of light, ammonia, and nicotinamide. Journal of Experimental Zoology. Part A, Ecological Genetics and Physiology, 319(7), 391-403. https://doi.org/10.1002/jez.1802

Cahill, C. M., Waterman, W. R., Xie, Y., Auron, P. E., \& Calderwood, S. K. (1996). Transcriptional repression of the prointerleukin 1beta gene by heat shock factor 1. Journal of Biological Chemistry, 271(40), 2487424879. https://www.ncbi.nlm.nih.gov/pubmed/8926278

Castillo, J., Teles, M., Mackenzie, S., \& Tort, L. (2009). Stress-related hormones modulate cytokine expression in the head kidney of gilthead seabream (Sparus aurata). Fish \& Shellfish Immunology, 27(3), 493-499. https://doi.org/10.1016/j.fsi.2009.06.021

Castro, R., Zou, J., Secombes, C. J., \& Martin, S. A. M. (2011). Cortisol modulates the induction of inflammatory gene expression in a rainbow trout macrophage cell line. Fish \& Shellfish Immunology, 30(1), 215-223. https://doi.org/10.1016/j.fsi.2010.10.010

Chao, D., He, X., Yang, Y., Balboni, G., Salvadori, S., Kim, D. H., \& Xia, Y. (2012). Hydrogen sulfide induced disruption of Na+ homeostasis in the cortex. Toxicological Sciences, 128(1), 198-208. https://doi.org/10.1093/toxsci/kfs125

Cheng, C.-H., Guo, Z.-X., Luo, S.-W., \& Wang, A.-L. (2018). Effects of high temperature on biochemical parameters, oxidative stress, DNA damage and apoptosis of pufferfish (Takifugu obscurus). Ecotoxicology and Environmental Safety, 150, 190-198. https://doi.org/10.1016/j.ecoenv.2017.12.045

Chivers, D. P., Dixson, D. L., White, J. R., McCormick, M. I., \& Ferrari, M. C. O. (2013). Degradation of chemical alarm cues and assessment of risk throughout the day. Ecology and Evolution, 3(11), 3925-3934. https://doi.org/10.1002/ece3.760

Cingi, S., Keinänen, M., \& Vuorinen, P. J. (2010). Elevated water temperature impairs fertilization and embryonic development of whitefish Coregonus lavaretus. Journal of Fish Biology, 76(3), 502-521. https://doi.org/10.1111/j.1095-8649.2009.02502.x

Clark, K. J., Boczek, N. J., \& Ekker, S. C. (2011). Stressing zebrafish for behavioral genetics. Reviews in the Neurosciences, 22(1), 49-62. https://doi.org/10.1515/RNS.2011.007

Colbert, P. L., Spencer, R.-J., \& Janzen, F. J. (2010). Mechanism and cost of synchronous hatching. Functional Ecology, 24(1), 112-121. https://doi.org/10.1111/j.1365-2435.2009.01602.x

Colinet, H., Sinclair, B. J., Vernon, P., \& Renault, D. (2015). Insects in fluctuating thermal environments. Annual Review of Entomology, 60(1), 123-140. https://doi.org/10.1146/annurev-ento-010814-021017 temperature exposure impairs emotional and cognitive responses and triggers dysregulation of neurodevelopment genes in fish. PeerJ, 7, e6338. https://doi.org/10.7717/peerj.6338 temperature has persistent, sexually dimorphic effects on zebrafish cardiac anatomy. Scientific Reports, 8(1), 8125. https://doi.org/10.1038/s41598-018-25991-8

Engeszer, R. E., Patterson, L. B., Rao, A. A., \& Parichy, D. M. (2007). Zebrafish in the wild: a review of natural 
history and new notes from the field. Zebrafish, 4(1), 21-40. https://doi.org/10.1089/zeb.2006.9997

Ferrari, M. C. O., Vavrek, M. A., Elvidge, C. K., \& Fridman, B. (2008). Sensory complementation and the acquisition of predator recognition by salmonid fishes. Behavioral Ecology and Sociobiology, 63(1), 113121.

Feugere, L., Angell, L., Fagents, J., Nightingale, R., Rowland, K., Skinner, S., Hardege, J. D., Bartels-Hardege, H. D., \& Wollenberg Valero, K. C. Behavioural stress feedback loops in benthic invertebrates caused by pH drop-induced metabolites. Manuscript Submitted for Publication.

Gau, P., Poon, J., Ufret-Vincenty, C., Snelson, C. D., Gordon, S. E., Raible, D. W., \& Dhaka, A. (2013). The zebrafish ortholog of TRPV1 is required for heat-induced locomotion. Journal of Neuroscience, 33(12), 5249-5260. https://doi.org/10.1523/JNEUROSCI.5403-12.2013

Giacomini, A. C. V. V., de Abreu, M. S., Koakoski, G., Idalêncio, R., Kalichak, F., Oliveira, T. A., da Rosa, J. G. S., Gusso, D., Piato, A. L., \& Barcellos, L. J. G. (2015). My stress, our stress: blunted cortisol response to stress in isolated housed zebrafish. Physiology \& Behavior, 139, 182-187. https://doi.org/10.1016/j.physbeh.2014.11.035

Giaquinto, P. C., \& Hoffmann, A. (2012). The scent of stress: Pintado catfish differentially respond to chemical cues from stressed conspecifics. Behaviour, 149(9), 941-951. https://doi.org/10.1163/1568539X-00003022

Goshen, I., \& Yirmiya, R. (2009). Interleukin-1 (IL-1): a central regulator of stress responses. Frontiers in Neuroendocrinology, 30(1), 30-45. https://doi.org/10.1016/j.yfrne.2008.10.001

Guo, L., Wang, Y., Liang, S., Lin, G., Chen, S., \& Yang, G. (2016). Tissue-overlapping response of half-smooth tongue sole (Cynoglossus semilaevis) to thermostressing based on transcriptome profiles. Gene, 586(1), $97-$ 104. https://doi.org/10.1016/j.gene.2016.04.020

Haesemeyer, M., Robson, D. N., Li, J. M., Schier, A. F., \& Engert, F. (2015). The structure and timescales of heat perception in larval zebrafish. Cell Systems, 1(5), 338-348. https://doi.org/10.1016/j.cels.2015.10.010

Haesemeyer, M., Robson, D. N., Li, J. M., Schier, A. F., \& Engert, F. (2018). A Brain-wide Circuit Model of Heat-Evoked Swimming Behavior in Larval Zebrafish. Neuron, 98(4), 817-831.e6. https://doi.org/10.1016/j.neuron.2018.04.013

Hallare, A. V., Schirling, M., Luckenbach, T., Köhler, H.-R., \& Triebskorn, R. (2005). Combined effects of temperature and cadmium on developmental parameters and biomarker responses in zebrafish (Danio rerio) embryos. Journal of Thermal Biology, 30(1), 7-17. https://doi.org/10.1016/j.jtherbio.2004.06.002

Hall, J. M., \& Warner, D. A. (2020a). Thermal sensitivity of lizard embryos indicates a mismatch between oxygen supply and demand at near-lethal temperatures. Journal of Experimental Zoology, 327, 466. https://doi.org/10.1002/jez.2359

Hall, J. M., \& Warner, D. A. (2020b). Ecologically relevant thermal fluctuations enhance offspring fitness: biological and methodological implications for studies of thermal developmental plasticity. Journal of Experimental Biology, 223(Pt 19). https://doi.org/10.1242/jeb.231902

Hazlett, B. A. (1985). Disturbance pheromones in the crayfishOrconectes virilis. Journal of Chemical Ecology, 
Henderson, L. J., Ryan, M. R., \& Rowland, H. M. (2017). Perch, Perca fluviatilis show a directional preference for, but do not increase attacks toward, prey in response to water-borne cortisol. PeerJ, 5(10), e3883. https://doi.org/10.7717/peerj.3883

Horsman, J. W., \& Miller, D. L. (2016). Mitochondrial Sulfide Quinone Oxidoreductase Prevents Activation of the Unfolded Protein Response in Hydrogen Sulfide. Journal of Biological Chemistry, 291(10), 5320-5325. https://doi.org/10.1074/jbc.M115.697102

Hosseini, S., Brenig, B., Tetens, J., \& Sharifi, A. R. (2019). Phenotypic plasticity induced using high ambient temperature during embryogenesis in domesticated zebrafish, Danio rerio. Reproduction in Domestic Animals, 54(3), 435-444. https://doi.org/10.1111/rda.13382

Hubbard, P. C., Barata, E. N., \& Canário, A. V. M. (2003). Olfactory sensitivity to catecholamines and their metabolites in the goldfish. Chemical Senses, 28(3), 207-218. https://doi.org/10.1093/chemse/28.3.207

Icoglu Aksakal, F., \& Ciltas, A. (2018). The impact of ultraviolet B (UV-B) radiation in combination with different temperatures in the early life stage of zebrafish (Danio rerio). Photochemical \& Photobiological Sciences, 17(1), 35-41. https://doi.org/10.1039/c7pp00236j

Ihaka, R., \& Gentleman, R. (1996). R: A Language for Data Analysis and Graphics. Journal of Computational and Graphical Statistics, 5(3), 299-314. https://doi.org/10.1080/10618600.1996.10474713

Jackson, M. R., Melideo, S. L., \& Jorns, M. S. (2012). Human sulfide:quinone oxidoreductase catalyzes the first step in hydrogen sulfide metabolism and produces a sulfane sulfur metabolite. Biochemistry, 51(34), 68046815. https://doi.org/10.1021/bi300778t

Jordão, L. C., \& Volpato, G. L. (2000). Chemical Transfer of Warning Information in Non-Injured Fish. Behaviour, 137(6), 681-690. http://www.jstor.org/stable/4535732

Kimmel, C. B., Ballard, W. W., Kimmel, S. R., Ullmann, B., \& Schilling, T. F. (1995). Stages of Embryonic Development of the Zebrafish. Developmental Dynamics, 203(3), 253-310.

Kimura, Y., Goto, Y.-I., \& Kimura, H. (2010). Hydrogen sulfide increases glutathione production and suppresses oxidative stress in mitochondria. Antioxidants \& Redox Signaling, 12(1), 1-13. https://doi.org/10.1089/ars.2008.2282

Kingsolver, J. G., Higgins, J. K., \& Augustine, K. E. (2015). Fluctuating temperatures and ectotherm growth: distinguishing non-linear and time-dependent effects. Journal of Experimental Biology, 218(Pt 14), 22182225. https://doi.org/10.1242/jeb.120733

Kirsten, K., Fior, D., Kreutz, L. C., \& Barcellos, L. J. G. (2018). First description of behavior and immune system relationship in fish. Scientific Reports, 8(1), 846. https://doi.org/10.1038/s41598-018-19276-3 
547

548

549

550

551

552

553

554

555

556

557

558

559

560

561

562

563

564

565

566

567

568

569

570

571

572

573

574

575

576

577

578

579

580

581

582

Lenhard, W., \& Lenhard, A. (2016). Calculation of effect sizes. Dettelbach (Germany): Psychometrica. https://doi.org/10.13140/RG.2.2.17823.92329

Lenth, R. (2019). emmeans: estimated marginal means, aka least-squares means. $R$ Package Version, 1(1), 3.

Lienart, G. D. H., Ferrari, M. C. O., \& McCormick, M. I. (2016). Thermal environment and nutritional condition affect the efficacy of chemical alarm cues produced by prey fish. Environmental Biology of Fishes, 99(10), 729-739. https://doi.org/10.1007/s10641-016-0516-7

Lim, M. Y.-T., Manzon, R. G., Somers, C. M., Boreham, D. R., \& Wilson, J. Y. (2017). The effects of fluctuating temperature regimes on the embryonic development of lake whitefish (Coregonus clupeaformis). Comparative Biochemistry and Physiology. Part A, Molecular \& Integrative Physiology, 214, 19-29. https://doi.org/10.1016/j.cbpa.2017.08.010

Liu, Z.-P., Gu, W.-B., Tu, D.-D., Zhu, Q.-H., Zhou, Y.-L., Wang, C., Wang, L.-Z., \& Shu, M.-A. (2018). Effects of both cold and heat stress on the liver of the giant spiny frog (Quasipaa spinosa): stress response and histological changes. Journal of Experimental Biology, 221(Pt 21). https://doi.org/10.1242/jeb.186379

Logan, C. A., \& Buckley, B. A. (2015). Transcriptomic responses to environmental temperature in eurythermal and stenothermal fishes. Journal of Experimental Biology, 218(Pt 12), 1915-1924. https://doi.org/10.1242/jeb.114397

Long, Y., Li, L., Li, Q., He, X., \& Cui, Z. (2012). Transcriptomic characterization of temperature stress responses in larval zebrafish. PloS One, 7(5), e37209. https://doi.org/10.1371/journal.pone.0037209

Long, Y., Yan, J., Song, G., Li, X., Li, X., Li, Q., \& Cui, Z. (2015). Transcriptional events co-regulated by hypoxia and cold stresses in Zebrafish larvae. BMC Genomics, 16(1), 385. https://doi.org/10.1186/s12864015-1560-y

López-Olmeda, J. F., \& Sánchez-Vázquez, F. J. (2011). Thermal biology of zebrafish (Danio rerio). Journal of Thermal Biology, 36(2), 91-104. https://doi.org/10.1016/j.jtherbio.2010.12.005

Lucon-Xiccato, T., Di Mauro, G., Bisazza, A., \& Bertolucci, C. (2020). Alarm cue-mediated response and learning in zebrafish larvae. Behavioural Brain Research, 380, 112446. https://doi.org/10.1016/j.bbr.2019.112446

Luna-Sánchez, M., Hidalgo-Gutiérrez, A., Hildebrandt, T. M., Chaves-Serrano, J., Barriocanal-Casado, E., Santos-Fandila, Á., Romero, M., Sayed, R. K., Duarte, J., Prokisch, H., Schuelke, M., Distelmaier, F., Escames, G., Acuña-Castroviejo, D., \& López, L. C. (2017). CoQ deficiency causes disruption of mitochondrial sulfide oxidation, a new pathomechanism associated with this syndrome. EMBO Molecular Medicine, 9(1), 78-95. https://doi.org/10.15252/emmm.201606345

Luu, I., Ikert, H., \& Craig, P. M. (2020). Chronic exposure to anthropogenic and climate related stressors alters transcriptional responses in the liver of zebrafish (Danio rerio) across multiple generations. Comparative Biochemistry and Physiology. Part C, Pharmacology, Toxicology \& Endocrinology, 240, 108918. https://doi.org/10.1016/j.cbpc.2020.108918

Lyu, L., Wen, H., Li, Y., Li, J., Zhao, J., Zhang, S., Song, M., \& Wang, X. (2018). Deep Transcriptomic 
Analysis of Black Rockfish (Sebastes schlegelii) Provides New Insights on Responses to Acute Temperature Stress. Scientific Reports, 8(1), 9113. https://doi.org/10.1038/s41598-018-27013-z

Madeira, D., Costa, P. M., Vinagre, C., \& Diniz, M. S. (2016). When warming hits harder: survival, cellular stress and thermal limits of Sparus aurata larvae under global change. Marine Biology, 163(4), 91.

Mahanty, A., Purohit, G. K., Banerjee, S., Karunakaran, D., Mohanty, S., \& Mohanty, B. P. (2016). Proteomic changes in the liver of Channa striatus in response to high temperature stress. Electrophoresis, 37(12), 1704-1717. https://doi.org/10.1002/elps.201500393

Mangiafico, S. (2018). rcompanion: Functions to support extension education program evaluation. $R$ Package Version, 1(2).

Mariana, S., \& Badr, G. (2019). Impact of heat stress on the immune response of fishes. Journal of Survey in Fisheries Sciences, 5(2), 149-159. https://doi.org/10.18331/SFS2019.5.2.14

Mathavan, S., Lee, S. G. P., Mak, A., Miller, L. D., Murthy, K. R. K., Govindarajan, K. R., Tong, Y., Wu, Y. L., Lam, S. H., Yang, H., Ruan, Y., Korzh, V., Gong, Z., Liu, E. T., \& Lufkin, T. (2005). Transcriptome analysis of zebrafish embryogenesis using microarrays. PLoS Genetics, 1(2), 260-276. https://doi.org/10.1371/journal.pgen.0010029

McGlashan, J. K., Spencer, R.-J., \& Old, J. M. (2012). Embryonic communication in the nest: metabolic responses of reptilian embryos to developmental rates of siblings. Proceedings of the Royal Society B: Biological Sciences, 279(1734), 1709-1715. https://doi.org/10.1098/rspb.2011.2074

Metz, J. R., Huising, M. O., Leon, K., Verburg-van Kemenade, B. M. L., \& Flik, G. (2006). Central and peripheral interleukin-1 $\beta$ and interleukin-1 receptor I expression and their role in the acute stress response of common carp, Cyprinus carpio L. Journal of Endocrinology, 191(1), 25-35. https://joe.bioscientifica.com/view/journals/joe/191/1/1910025.xml

Morgan, I. J., McDonald, D. G., \& Wood, C. M. (2001). The cost of living for freshwater fish in a warmer, more polluted world. Global Change Biology, 7(4), 345-355.

Morgan, R., Sundin, J., Finnøen, M. H., Dresler, G., Vendrell, M. M., Dey, A., Sarkar, K., \& Jutfelt, F. (2019). Are model organisms representative for climate change research? Testing thermal tolerance in wild and laboratory zebrafish populations. Conservation Physiology, 7(1), coz036. https://doi.org/10.1093/conphys/coz036

Oulton, L. J., Haviland, V., \& Brown, C. (2013). Predator Recognition in Rainbowfish, Melanotaenia duboulayi, Embryos. PLos One, 8(10), e76061. https://doi.org/10.1371/journal.pone.0076061

Paaijmans, K. P., Heinig, R. L., Seliga, R. A., Blanford, J. I., Blanford, S., Murdock, C. C., \& Thomas, M. B. (2013). Temperature variation makes ectotherms more sensitive to climate change. Global Change Biology, 19(8), 2373-2380. https://doi.org/10.1111/gcb.12240

Peterson, R. A., \& Cavanaugh, J. E. (2020). Ordered quantile normalization: a semiparametric transformation built for the cross-validation era. Journal of Applied Statistics, 47(13-15), 2312-2327. https://doi.org/10.1080/02664763.2019.1630372 
619

620

621

622

623

624

625

626

627

628

629

630

631

632

633

634

635

636

637

638

639

640

641

642

643

644

645

646

647

648

649

650

651

652

653

654

Poisson, A., Valotaire, C., Borel, F., Bertin, A., Darmaillacq, A.-S., Dickel, L., \& Colson, V. (2017). Embryonic exposure to a conspecific alarm cue triggers behavioural plasticity in juvenile rainbow trout. Animal Behaviour, 133, 35-45. https://doi.org/10.1016/j.anbehav.2017.09.013

Pörtner, H. O., \& Peck, M. A. (2010). Climate change effects on fishes and fisheries: towards a cause-and-effect understanding. Journal of Fish Biology, 77(8), 1745-1779. https://doi.org/10.1111/j.10958649.2010.02783.x

Pörtner, H. O., Roberts, D. C., Masson-Delmotte, V., Zhai, P., Tignor, M., Poloczanska, E., Mintenbeck, K., Nicolai, M., Okem, A., Petzold, J., \& Others. (2019). IPCC, 2019: Summary for Policymakers. IPCC Special Report on the Ocean and Cryosphere in a Changing Climate.

Pype, C., Verbueken, E., Saad, M. A., Casteleyn, C. R., Van Ginneken, C. J., Knapen, D., \& Van Cruchten, S. J. (2015). Incubation at $32.5^{\circ} \mathrm{C}$ and above causes malformations in the zebrafish embryo. Reproductive Toxicology, 56, 56-63. https://doi.org/10.1016/j.reprotox.2015.05.006

Quinzii, C. M., Luna-Sanchez, M., Ziosi, M., Hidalgo-Gutierrez, A., Kleiner, G., \& Lopez, L. C. (2017). The Role of Sulfide Oxidation Impairment in the Pathogenesis of Primary CoQ Deficiency. Frontiers in Physiology, 8, 525. https://doi.org/10.3389/fphys.2017.00525

Ribas, L., Liew, W. C., Díaz, N., Sreenivasan, R., Orbán, L., \& Piferrer, F. (2017). Heat-induced masculinization in domesticated zebrafish is family-specific and yields a set of different gonadal transcriptomes.

Proceedings of the National Academy of Sciences, 114(6), E941-E950. https://doi.org/10.1073/pnas.1609411114

Ritchie, M. E., Phipson, B., Wu, D., Hu, Y., Law, C. W., Shi, W., \& Smyth, G. K. (2015). limma powers differential expression analyses for RNA-sequencing and microarray studies. Nucleic Acids Research, 43(7), e47. https://doi.org/10.1093/nar/gkv007

Roggatz, C. C., Fletcher, N., Benoit, D. M., Algar, A. C., Doroff, A., Wright, B., Wollenberg Valero, K. C., \& Hardege, J. D. (2019). Saxitoxin and tetrodotoxin bioavailability increases in future oceans. Nature Climate Change, 9(11), 840-844. https://doi.org/10.1038/s41558-019-0589-3

Rose, P., Moore, P. K., \& Zhu, Y. Z. (2017). H2S biosynthesis and catabolism: new insights from molecular studies. In Cellular and Molecular Life Sciences (Vol. 74, Issue 8, pp. 1391-1412). https://doi.org/10.1007/s00018-016-2406-8

RStudio Team. (2020). RStudio: Integrated Development Environment for R. RStudio, PBC. http://www.rstudio.com/

Sawilowsky, S. S. (2009). New Effect Size Rules of Thumb. Journal of Modern Applied Statistical Methods: JMASM, 8(2), 26. https://doi.org/10.22237/jmasm/1257035100

Saxon, A. D., O’Brien, E. K., \& Bridle, J. R. (2018). Temperature fluctuations during development reduce male fitness and may limit adaptive potential in tropical rainforest Drosophila. Journal of Evolutionary Biology, 31(3), 405-415. https://doi.org/10.1111/jeb.13231

Schaefer, J., \& Ryan, A. (2006). Developmental plasticity in the thermal tolerance of zebrafish Danio rerio. 
Journal of Fish Biology, 69(3), 722-734. https://doi.org/10.1111/j.1095-8649.2006.01145.x

656

657

658

659

660

661

662

663

664

665

666

667

668

669

670

671

672

673

674

675

676

677

678

679

680

681

682

683

684

685

686

687

688

689

690

Schirone, R. C., \& Gross, L. (1968). Effect of temperature on early embryological development of the zebra fish, Brachydanio rerio. Journal of Experimental Zoology, 169(1), 43-52. https://doi.org/10.1002/jez.1401690106

Schmidt, K., \& Starck, J. M. (2010). Developmental plasticity, modularity, and heterochrony during the phylotypic stage of the zebra fish, Danio rerio. Journal of Experimental Zoology. Part B, Molecular and Developmental Evolution, 314(2), 166-178. https://doi.org/10.1002/jez.b.21320

Schnurr, M. E., Yin, Y., \& Scott, G. R. (2014). Temperature during embryonic development has persistent effects on metabolic enzymes in the muscle of zebrafish. Journal of Experimental Biology, 217(Pt 8), 13701380. https://doi.org/10.1242/jeb.094037

Schröter, C., Herrgen, L., Cardona, A., Brouhard, G. J., Feldman, B., \& Oates, A. C. (2008). Dynamics of zebrafish somitogenesis. Developmental Dynamics: An Official Publication of the American Association of Anatomists, 237(3), 545-553. https://doi.org/10.1002/dvdy.21458

Scott, G. R., \& Johnston, I. A. (2012). Temperature during embryonic development has persistent effects on thermal acclimation capacity in zebrafish. Proceedings of the National Academy of Sciences, 109(35), 14247-14252. https://doi.org/10.1073/pnas.1205012109

Simčič, T., Jesenšek, D., \& Brancelj, A. (2015). Effects of increased temperature on metabolic activity and oxidative stress in the first life stages of marble trout (Salmo marmoratus). Fish Physiology and Biochemistry, 41(4), 1005-1014. https://doi.org/10.1007/s10695-015-0065-6

Skjærven, K. H., Hamre, K., Penglase, S., Finn, R. N., \& Olsvik, P. A. (2014). Thermal stress alters expression of genes involved in one carbon and DNA methylation pathways in Atlantic cod embryos. Comparative Biochemistry and Physiology. Part A, Molecular \& Integrative Physiology, 173C, 17-27. https://doi.org/10.1016/j.cbpa.2014.03.003

Somero, G. N. (2010). The physiology of climate change: how potentials for acclimatization and genetic adaptation will determine "winners" and "losers." Journal of Experimental Biology, 213(6), 912-920. https://doi.org/10.1242/jeb.037473

Spence, R., Gerlach, G., Lawrence, C., \& Smith, C. (2008). The behaviour and ecology of the zebrafish, Danio rerio. Biological Reviews of the Cambridge Philosophical Society, 83(1), 13-34. https://doi.org/10.1111/j.1469-185X.2007.00030.x

Strecker, R., Seiler, T.-B., Hollert, H., \& Braunbeck, T. (2011). Oxygen requirements of zebrafish (Danio rerio) embryos in embryo toxicity tests with environmental samples. Comparative Biochemistry and Physiology. Toxicology \& Pharmacology: CBP, 153(3), 318-327. https://doi.org/10.1016/j.cbpc.2010.12.002

Toa, D. G., Afonso, L. O. B., \& Iwama, G. K. (2004). Stress response of juvenile rainbow trout (Oncorhynchus mykiss) to chemical cues released from stressed conspecifics. Fish Physiology and Biochemistry, 30(2), 103-108. https://doi.org/10.1007/s10695-005-0266-5

Torchiano, M. (2016). effsize: efficient effect size computation. $R$ Package. 
691

692

693

694

695

696

697

698

699

700

701

702

703

704

705

706

707

708

709

710

711

712

713

714

715

716

717

718

719

720

721

722

723

724

725

726

Vasseur, D. A., DeLong, J. P., Gilbert, B., Greig, H. S., Harley, C. D. G., McCann, K. S., Savage, V., Tunney, T. D., \& O'Connor, M. I. (2014). Increased temperature variation poses a greater risk to species than climate warming. Proceedings of the Royal Society B: Biological Sciences, 281(1779), 20132612. https://doi.org/10.1098/rspb.2013.2612

Villamizar, N., Ribas, L., Piferrer, F., Vera, L. M., \& Sánchez-Vázquez, F. J. (2012). Impact of daily thermocycles on hatching rhythms, larval performance and sex differentiation of zebrafish. PloS One, 7(12), e52153. https://doi.org/10.1371/journal.pone.0052153

Villamizar, N., Vera, L. M., Foulkes, N. S., \& Sánchez-Vázquez, F. J. (2014). Effect of lighting conditions on zebrafish growth and development. Zebrafish, 11(2), 173-181. https://doi.org/10.1089/zeb.2013.0926

Vinagre, C., Madeira, D., Narciso, L., Cabral, H. N., \& Diniz, M. (2012). Effect of temperature on oxidative stress in fish: Lipid peroxidation and catalase activity in the muscle of juvenile seabass, Dicentrarchus labrax. Ecological Indicators, 23, 274-279. https://doi.org/10.1016/j.ecolind.2012.04.009

Vitkovic, L., Bockaert, J., \& Jacque, C. (2000). "Inflammatory” cytokines: neuromodulators in normal brain? Journal of Neurochemistry, 74(2), 457-471. https://doi.org/10.1046/j.1471-4159.2000.740457.x

Wang, Y., Liu, Z., Li, Z., Shi, H., Kang, Y., Wang, J., Huang, J., \& Jiang, L. (2016). Effects of heat stress on respiratory burst, oxidative damage and SERPINH1 (HSP47) mRNA expression in rainbow trout Oncorhynchus mykiss. Fish Physiology and Biochemistry, 42(2), 701-710. https://doi.org/10.1007/s10695015-0170-6

Whitehouse, L. M., McDougall, C. S., Stefanovic, D. I., Boreham, D. R., Somers, C. M., Wilson, J. Y., \& Manzon, R. G. (2017). Development of the embryonic heat shock response and the impact of repeated thermal stress in early stage lake whitefish (Coregonus clupeaformis) embryos. Journal of Thermal Biology, 69, 294-301. https://doi.org/10.1016/j.jtherbio.2017.08.013

Wollenberg Valero, K. C., Garcia-Porta, J., Irisarri, I., Feugere, L., Bates, A., Kirchhof, S., Glavaš, O. J., Pafilis, P., Samuel, S. F., Müller, J., Vences, M., Turner, A. P., Beltran-Alvarez, P., \& Storey, K. B. (2019). Abiotic environmental adaptation in vertebrates is characterized by functional genomic constraint. In bioRxiv (p. 726240). https://doi.org/10.1101/726240

Xia, J. H., Li, H. L., Li, B. J., Gu, X. H., \& Lin, H. R. (2018). Acute hypoxia stress induced abundant differential expression genes and alternative splicing events in heart of tilapia. Gene, 639, 52-61. https://doi.org/10.1016/j.gene.2017.10.002

Xia, J., Peng, M., Huang, Y., \& Elvidge, C. K. (2020). Acute warming in winter eliminates chemical alarm responses in threatened Qinling lenok Brachymystax lenok tsinlingensis. Science of the Total Environment, 142807. https://doi.org/10.1016/j.scitotenv.2020.142807

Yokogawa, T., Iadarola, M., \& Burgess, H. (2014). Thermal response behaviors in larval zebrafish: startle escape, thermotaxis and thermal arousal. Measuring Behavior 2014.

Yonezawa, D., Sekiguchi, F., Miyamoto, M., Taniguchi, E., Honjo, M., Masuko, T., Nishikawa, H., \& Kawabata, A. (2007). A protective role of hydrogen sulfide against oxidative stress in rat gastric mucosal 
epithelium. Toxicology, 241(1-2), 11-18. https://doi.org/10.1016/j.tox.2007.07.020

728

729

730

731

732

733

734

735

736

737

738

739

740

Zahangir, M. M., Haque, F., Mustakim, G. M., Khatun, H., \& Islam, M. S. (2015). Effect of water pH on the early developmental responses in zebrafish (danio rerio). Progressive Agriculture, 26(1), 85-89. https://doi.org/10.3329/pa.v26i1.24521

Zhang, Q., Kopp, M., Babiak, I., \& Fernandes, J. M. O. (2018). Low incubation temperature during early development negatively affects survival and related innate immune processes in zebrafish larvae exposed to lipopolysaccharide. Scientific Reports, 8(1), 4142. https://doi.org/10.1038/s41598-018-22288-8

Zhuang, Z., Xu, L., Yang, J., Gao, H., Zhang, L., Gao, X., Li, J., \& Zhu, B. (2020). Weighted Single-Step Genome-Wide Association Study for Growth Traits in Chinese Simmental Beef Cattle. Genes, 11(2). https://doi.org/10.3390/genes11020189

Ziosi, M., Di Meo, I., Kleiner, G., Gao, X.-H., Barca, E., Sanchez-Quintero, M. J., Tadesse, S., Jiang, H., Qiao, C., Rodenburg, R. J., Scalais, E., Schuelke, M., Willard, B., Hatzoglou, M., Tiranti, V., \& Quinzii, C. M. (2017). Coenzyme Q deficiency causes impairment of the sulfide oxidation pathway. EMBO Molecular Medicine, 9(1), 96-111. https://doi.org/10.15252/emmm.201606356 
bioRxiv preprint doi: https://doi.org/10.1101/2021.04.07.438623; this version posted April 8, 2021. The copyright holder for this preprint (which

was not certified by peer review) is the author/funder, who has granted bioRxiv a license to display the preprint in perpetuity. It is made available under aCC-BY-ND 4.0 International license.

\section{Tables}

742 Table 1. Effects of thermal stress and stress medium on the growth index of pre-hatching zebrafish embryos.

743 A two-way ANOVA was used to test the effects of the predictors (thermal stress and stress medium) on changes

744 in growth index. Effect size is computed as Cohen's $|\mathrm{d}|$ and interpreted according to thresholds given in 745 Sawilowsky (Sawilowsky, 2009). Effect sizes of significant p-values $(\mathrm{P} \leq 0.05)$ are shown in bold.

746

\begin{tabular}{lllll}
\hline Model terms & F statistic & P & $\mid \mathbf{d}$ & Effect size \\
\hline Stress Medium & 6.291 & 0.0128 & 0.29 & small \\
Thermal Stress & 75.502 & 0.0001 & 1.14 & large \\
Thermal Stress x Stress Medium & 7.498 & 0.0067 & 1.27 & very large \\
\hline
\end{tabular}


748 Table 2. Effects of thermal stress and stress medium on the burst activity percentage of pre-hatching

749 zebrafish embryos. A two-way Scheirer-Ray-Hare test was used to test the effects of the predictors (thermal stress

750 and stress medium) on burst activity percentages across treatments. Effect size is computed as Cohen's $|\mathrm{d}|$ and

751 interpreted according to thresholds given in Sawilowsky (Sawilowsky 2009). Effect sizes of significant p-values

$752 \quad(\mathrm{P} \leq 0.05)$ are shown in bold.

753

\begin{tabular}{lllll}
\hline Model terms & H statistic & P & $\mid \mathbf{d}$ & Effect size \\
\hline Stress Medium & 9.3222 & 0.0023 & 0.49 & small \\
Thermal Stress & 17.0080 & 0.0001 & 0.55 & medium \\
Thermal Stress x Stress Medium & 1.8193 & 0.1774 & 0.99 & large \\
\hline
\end{tabular}

754 
Table 3. Effect of thermal stress and stress medium on gene expression of pre-hatching zebrafish embryos.

756 Effects of model terms (thermal stress and stress medium) on the expression of IL-1 $\beta$, SOD1 and SQOR obtained

757 via moderated t-tests (t-statistic) with lmfit and eBayes in the limma $\mathrm{R}$ package. B describes the log-odds of gene 758 expression. Effect size is computed as Cohen's $|\mathrm{d}|$ and interpreted according to thresholds given in Sawilowsky 759 (Sawilowsky 2009). Effect sizes of significant $\mathrm{p}$-values $(\mathrm{P} \leq 0.05)$ are shown in bold.

760

\begin{tabular}{|c|c|c|c|c|c|c|}
\hline Model & erms & $\mathbf{t}$ & B & $\mathbf{P}$ & $|\mathbf{d}|$ & Effect size \\
\hline$\overline{\text { IL-1 } \beta}$ & & & & & & \\
\hline & Thermal Stress & 1.03 & -4.70 & 0.310 & 0.49 & medium \\
\hline & Stress Medium & 2.28 & -3.95 & 0.038 & 1.2 & very large \\
\hline SOD1 & & & & & & \\
\hline & Thermal Stress & 1.65 & -4.27 & 0.108 & 1.08 & large \\
\hline & Stress Medium & 0.32 & -6.16 & 0.757 & 0.17 & small \\
\hline SQOR & & & & & & \\
\hline & Thermal Stress & -0.17 & -4.99 & 0.869 & 0.11 & small \\
\hline & Stress Medium & -3.54 & -1.67 & 0.003 & 2.43 & huge \\
\hline
\end{tabular}




\section{Figures}

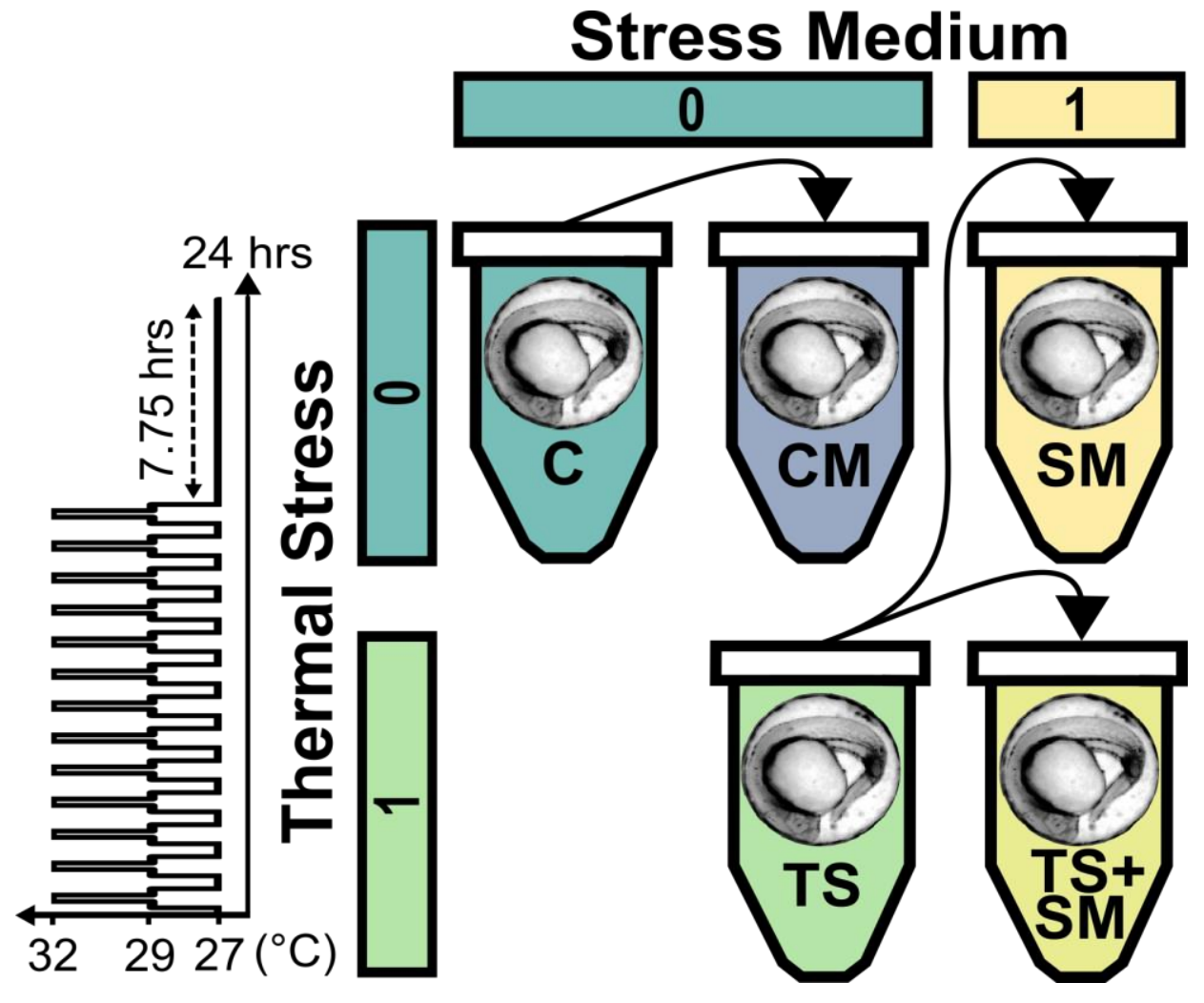

Figure 1. Overview of experimental design. In a two-way factorial design (represented by 0 and 1 for factor levels, respectively), zebrafish embryos (Danio rerio) were exposed to either fluctuating thermal stress (inset diagram) followed by a recovery period (dashed arrow line) or constant control temperature $\left(27^{\circ} \mathrm{C}\right)$, in combination with fresh E3 medium or stress medium containing putative stress metabolites produced by one previously thermally-stressed embryo. An additional treatment was incubated at $27^{\circ} \mathrm{C}$ in a control medium containing metabolites from an embryo previously exposed to control conditions. Plain black arrows indicate medium transfer in which a new embryo was incubated. CM: control medium, C: control, SM: stress medium, TS: thermal stress, TS+SM: thermal stress + stress medium. 

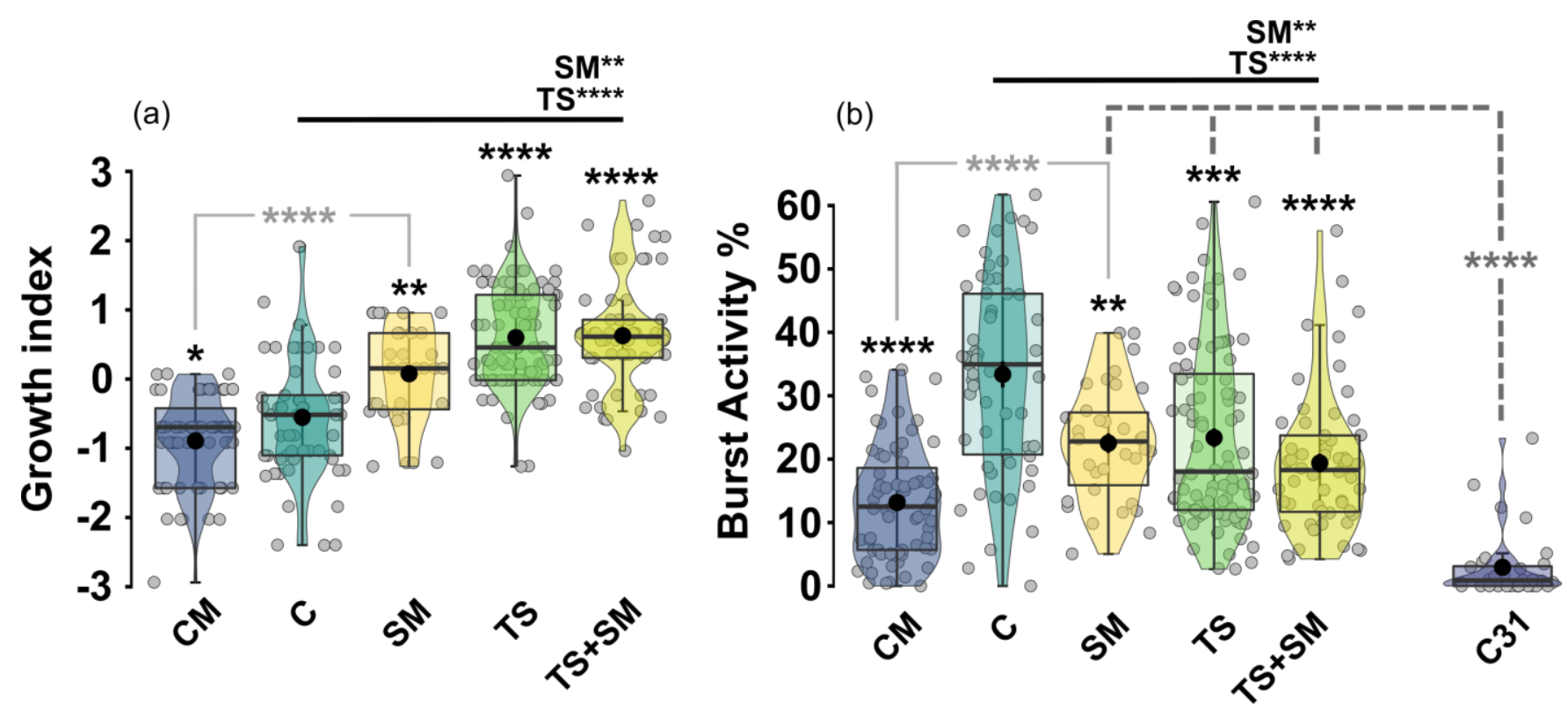

Figure 2. Thermal stress and stress medium accelerate the growth index (a) and lower the burst activity percentage (b) of pre-hatching zebrafish embryos. CM: control medium $(n=67)$, C: control $(n=57)$, SM: stress medium $(n=34)$, TS: thermal stress $(n=90), \mathrm{TS}+\mathrm{SM}$ : thermal stress + stress medium $(n=56), \mathrm{C} 31$ : control after 31 hrs of incubation (reaching prim-6 stage). Boxes represent median, 25\%-75\% quartiles, and whiskers are minimum and maximum values within 1.5 IQR (interquartile range). Grey dots represent individual data points and black dots represent mean values. Effects of predictors (thermal stress and stress medium) were tested with two-factorial tests. Significant predictors are indicated in bold above horizontal black lines with *: $P \leq 0.05, * *: P$ $\leq 0.01, * * *: P \leq 0.001, * * *: P \leq 0.0001$. Significance of pairwise post-hoc tests is likewise indicated in asterisks, for treatments against C (bold black), between SM and CM (light grey), or between over-developing treatments (SM, TS, TS+SM) and C31 (dashed bar with dark grey asterisk). 
(a)

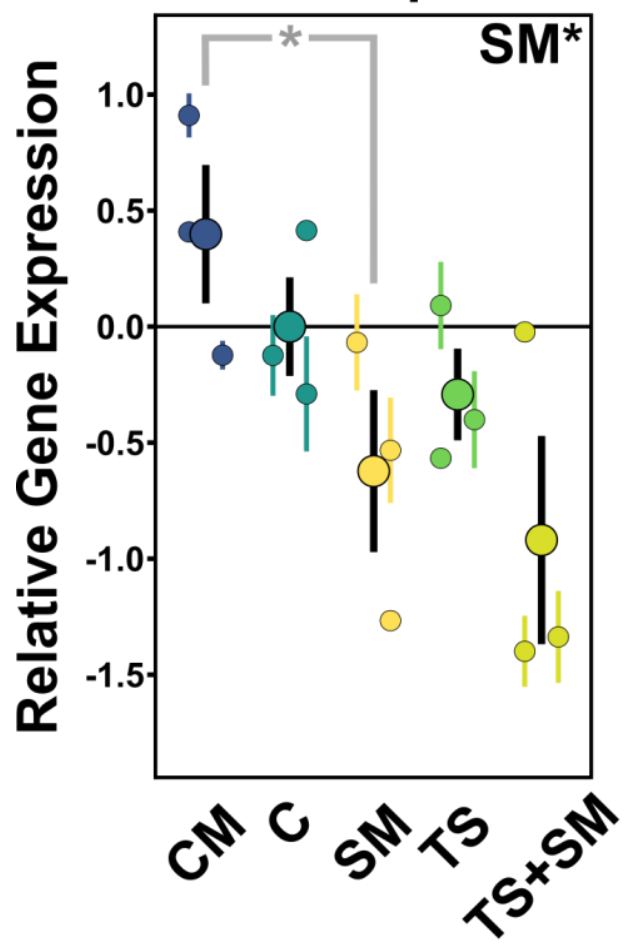

(b)

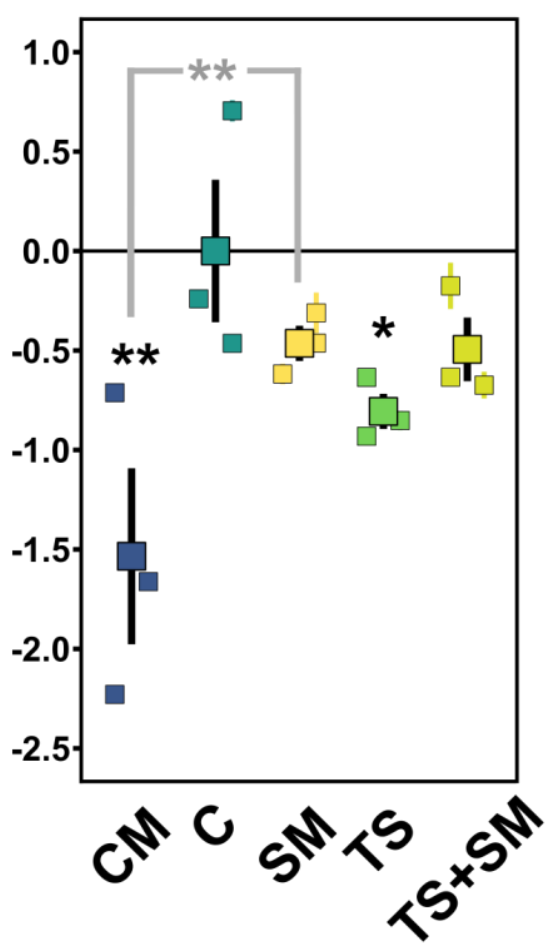

(c) SQOR

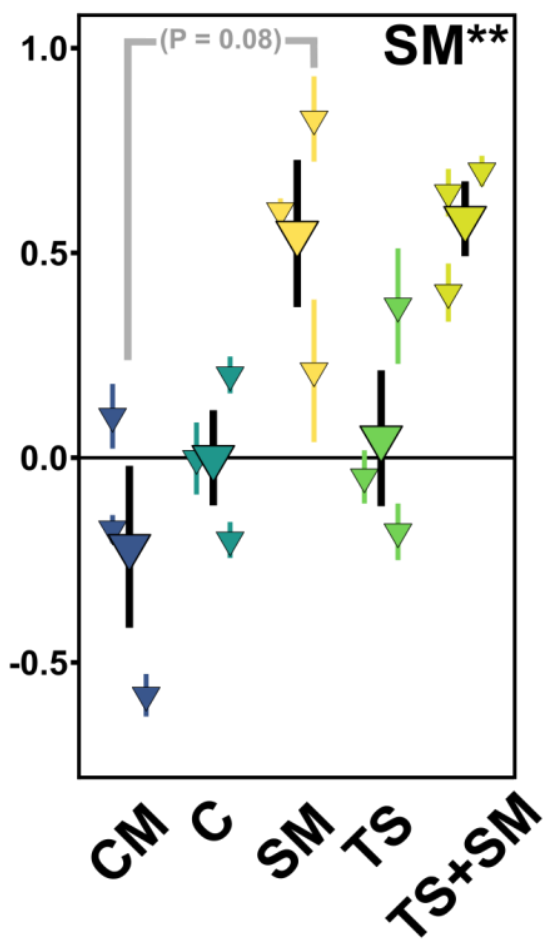

Figure 3. Stress medium alters stress-related gene expression of zebrafish embryos. (a) IL-1 $\beta$ (interleukin-

$1 \beta$ ), involved in immune response. (b) SOD1 (superoxide dismutase 1), involved in antioxidant response. (c) SQOR (sulfide:quinone oxidoreductase), involved in metabolism and antioxidant response. Jittered symbols represent mean $\log _{2} 2^{-\Delta \Delta C T} \pm S E$ (coloured bars) values of each biological replicate. Black symbols represent mean $\log _{2} 2^{-\Delta \Delta C T} \pm$ SE (black bars) values of each biological treatment. Expression is relative to control C (mean values as black horizontal lines). CM: control medium, C: control, SM: stress medium, TS: thermal stress, TS+SM: thermal stress + stress medium. Annotations on top right corners represent the factorial effects of thermal stress (TS) and stress medium (SM) on C, SM, TS, TS+SM. Black asterisks above each mean value only show the pairwise comparisons against the control C. Comparisons between CM and SM are represented in grey by asterisks and dashed horizontal lines. Statistics were computed using moderated t-tests (Limma, Bioconductor, R) with Bonferroni adjustment with *: $\mathrm{P} \leq 0.05, * *: \mathrm{P} \leq 0.01$. Sample size: $n=3$ biological replicates for each treatment, with 60 pooled embryos per each replicate. 